\title{
Negative priming effects that are bigger than a breadbox: Attention to distractors does not eliminate negative priming, it enhances it
}

\author{
PENNY A. MACDONALD and STEVE JOORDENS \\ University of Toronto, Scarborough, Ontario, Canada \\ and \\ KEN N. SEERGOBIN \\ McMaster University, Hamilton, Ontario, Canada
}

\begin{abstract}
In a series of experiments, we examined the effect of requiring subjects to attend to distractors in a test of negative priming. This was accomplished by using a referent size-selection task in which subjects were instructed to name the larger animal and to ignore the smaller animal in a word pair. The result was a quadrupling of the standard negative priming effect, suggesting that negative priming not only occurs for attended distractors, it is actually enhanced. We demonstrated that this enhancement of the effect was not due solely to increased latencies in the referent size-selection task, because neither decreasing base response times in other referent size-selection tasks nor increasing base response times in typical color-selection tasks substantially affected the respective negative priming effects. Although these findings can be accommodated within current theories of negative priming, they challenge the basic assumption that the negative priming effect arises because the critical item was ignored or not attended to on the prime trial.
\end{abstract}

Efficiently selecting and responding to targets while disregarding distractors is an incredibly adaptive ability. This explains the increasing interest in the negative priming phenomenon that has generally been attributed to such processes (Neill, 1977; Tipper, 1985). A typical negative priming study requires selection of a target item from among one or more distractors on a first trial (i.e., the prime trial). The task is then normally repeated on a second trial (i.e., the probe trial). The critical element is the relation between the targets and the distractors on the consecutive prime and probe trials. In the control condition, no relation exists between the targets and the distractors on the two consecutive trials. That is, different, unrelated distractors and targets occur on the prime and the probe trials. This condition serves as a baseline in terms of latency and error rate for performing the task. The ignored repetition condition is the crucial experimental condition. The manipulation in this condition consists of presenting as a target, on the probe trial, the item that served as the ignored distractor on the prime trial. In this condition, responses to the probe target are normally slower and more error prone, relative to the control condition: An em-

This work was supported by a Natural Sciences and Engineering Research Council of Canada (NSERC) Individual Research Grant awarded to the second author. We thank Colin MacLeod for reviewing earlier drafts of this manuscript. Correspondence concerning this article should be addressed to P. A. MacDonald, Division of Life Sciences, University of Toronto at Scarborough, 1265 Military Trail, Scarborough, ON MIC 1A4, Canada (e-mail: penny@psych.utoronto.ca). pirical phenomenon termed negative priming (DalrympleAlford \& Budayr, 1966).

Reviews of the negative priming literature by May, Kane, and Hasher (1995) and Fox (1995) begin by claiming that because we are constantly bombarded by a myriad of both relevant and irrelevant information, we can not attend to all stimuli. The claim is that, because of limitations in processing capacity, we must selectively attend to the important aspects of our environment and ignore that which does not serve an immediate purpose. Fox states that "a central aspect of coherent perceptual-motor processes is the ability to select and respond to relevant objects and the ability to successfully ignore irrelevant objects. The nature of the mechanisms underlying this visual selective attention is of enduring interest to cognitive psychologists" (p. 145). The implication is that this process of selective attention produces negative priming when an item that was selected against becomes a target item on the subsequent trial.

Relating negative priming to selective attention, however, is not straightforward, because both early- and lateselection accounts of attention exist. According to earlyselection accounts (e.g., Broadbent, 1958), only relevant information consistently receives deep processing, whereas most irrelevant information receives only cursory processing, focusing on perceptual characteristics. According to late-selection accounts (Deutsch \& Deutsch, 1963), however, both relevant and irrelevant information undergoes deep processing, although only selected information reaches phenomenal awareness. When negative priming has been related to processes of selective attention, few 
authors have clarified the version of selective attention to which they subscribe.

The bulk of the negative priming data appears to be consistent with a late-selection model of attention. Many studies show that negative priming occurs despite changes in the physical form of the critical stimulus from the prime to the probe (e.g., Dalrymple-Alford \& Budayr, 1966; Driver \& Baylis, 1993; Tipper \& Driver, 1988). This suggests that distractors are processed beyond physical features, to the level of a more abstract, central representation. Negative priming also persists across differing response modalities (Neill, Lissner, \& Beck, 1990; Tipper, MacQueen, \& Brehaut, 1988), again suggesting that distractors are processed beyond physical features.

Tipper and Driver (1988) provided additional support for a late-selection explanation of negative priming. They found that negative priming occurred for semantically related items as well as for repeated items. In their experiment, subjects were shown pairs of line drawings and were asked to name the red picture. They were slower to name a picture of a dog if they had ignored a picture of a cat on the preceding prime trial. This finding bolsters the claim that negative priming results from a late-selection mechanism of attention, because the items corresponded to one another primarily in terms of categorical features. The distractor must have been processed at least to the level of semantic analysis.

Not all the empirical evidence supports the link between negative priming and a late-selection model of attention. Chiappe and MacLeod (1995) performed an experiment analogous to that of Tipper and Driver (1988), using words instead of pictures. They obtained negative priming only when the critical item was identical on the prime and the probe. Negative priming failed to occur for semantically related stimuli. Others also have not achieved associative negative priming (Tipper \& Baylis, 1987, Experiment 2; Yee, 1991, Experiment 1 for one ignored word and Experiment 2). The findings with words are consistent with an early-selection account of attention, if, indeed, negative priming results from selective attention.

Although much of the literature is consistent with selective attention views of negative priming, recent evidence challenges this relation. Milliken and Joordens (1996) conducted a negative priming experiment, presenting two items on both the prime and the probe trials. In contrast to the typical negative priming procedure, however, the prime stimuli only appeared for $100 \mathrm{msec}$, and the subjects were not asked to respond overtly to either item. Negative priming persisted, despite the fact that subjects were not required to selectively attend to one item and to ignore the other on the prime trial. These findings cast doubt on the prevalent view that negative priming is a consequence of selective attention on the prime.

In a second series of experiments, Milliken, Joordens, Merikle, and Seiffert (1998) provided another demonstration of negative priming in the absence of selection on the prime trial. Specifically, the procedure involved briefly presenting (i.e., for $43 \mathrm{msec}$ ), then masking, a single prime item followed by a typical two-item probe display. Responses on the probe trial were slower to targets that matched the briefly presented prime word, relative to targets that were not previously presented (i.e., negative priming). This finding further challenges the notion that negative priming results from selective attention processes on the prime trial. It is unclear why selective attention would be operating in a single-item prime situation.

Although these manipulations broaden the scope of situations that yield negative priming, the results do not completely negate the link between negative priming and selective attention. The possibility remains that the subjects ignored the prime trial entirely and that, consequently, prime items were selected against or processed without attention. Given that the subjects were not required to respond on prime trials and that prime items were displayed very briefly, this speculation is plausible. In fact, another experiment in the Milliken et al. (1998) study provided results consistent with this possibility. When single prime words were presented for a slightly longer duration (i.e., $200 \mathrm{msec}$ ), the instruction to attend to or to ignore these items determined their effect on subsequent probe processing. Subjects who were instructed to attend to prime words showed positive priming, whereas subjects who were instructed to ignore them revealed negative priming in the repeated-item condition. Thus, these manipulations failed to ensure that the critical prime items were not blocked from attention. Because this is the aspect of selective attention that purportedly produces negative priming (Fox, 1995; May et al., 1995), these results are not entirely inconsistent with selective attention accounts of negative priming.

In summary, the current evidence concerning the relation between negative priming and selective attention is ambiguous. Some findings seem consistent with an early-selection view of attention (Chiappe \& MacLeod, 1995), whereas others support the relation between negative priming and late-selection theories (Tipper \& Driver, 1988). More recent studies challenge the link between negative priming and selective attention altogether (Milliken \& Joordens, 1996; Milliken et al., 1998). We believed that this issue needed to be addressed more directly.

The goal of the present study was to investigate the relation between selective attention and negative priming more directly. The strategy we employed stood in contrast to that of Milliken and Joordens (1996) and Milliken et al. (1998). Rather than create a situation in which neither the target nor the distractor was selectively attended to on the prime trial, we created a situation in which both the target and the distractor were attended to on the prime and probe trials. If negative priming is produced by a mechanism of selective attention (either early or late), the effect should be contingent on limiting the processing of distractors and blocking them from phenomenal awareness on ignored repetition trials. That is, if negative priming is indeed the consequence of having ignored or processed critical items without attention on the prime trial, 
it should not result when subjects are required to attend to both targets and distractors.

\section{EXPERIMENT 1A}

The primary objective of Experiment $1 \mathrm{~A}$ was to compare the results obtained in a typical negative priming procedure with those achieved in a variant of the procedure that required subjects to attend to both the targets and the distractors prior to selection. We asked the subjects to read the name of the larger animal in a stimulus pair in Experiments 1A and 1B. To make this referent size judgment, the subjects needed to attend to and process semantic characteristics of both the target and the distractor before selecting and responding to the target. In this manner, we were able to control the depth to which targets and, more importantly, distractors were processed. In the more conventional task of Experiments $1 \mathrm{~A}$ and $1 \mathrm{~B}$, we asked the subjects to read the word that appeared in red. As is typical in this case, the subjects needed only to identify and select the red word, independently of any other feature of the distractor.

\section{Method}

Subjects. Twenty volunteers from the introductory psychology class at the University of Toronto at Scarborough took part in the experiment in exchange for bonus credit. All the subjects had normal or corrected-to-normal vision. All the subjects were sufficiently fluent in English to perform the task without difficulty.

Procedure. On each display (i.e., the prime display and the probe display), the names of two animals appeared in the center of the computer screen. One name was in white, the other was in red. In one block of trials, the subjects were asked to read the animal name printed in red. This color-selection task is a typical negative priming procedure. In the other block, the subjects were instructed to name the larger animal in the pair, with size defined relative to a preset continuum. This referent size-selection task required a size comparison of the referents of the two animal names before selection and responding could occur. The assumption was that subjects had to attend to and process to the level of semantics both the target and the distractor to make this judgment, on every trial.

Each trial consisted of both a prime and a probe display and proceeded as follows: (1) A focal point consisting of four plus signs appeared in the center of the screen for $250 \mathrm{msec}$; (2) a blank screen was presented for $250 \mathrm{msec} ;(3)$ the prime words were visible until the subject responded; (4) a mask (i.e., \&\&\&\&\&\&) appeared for $250 \mathrm{msec}$; and (5) the probe words appeared until the subject responded. The prime and probe stimuli remained in the center of the screen until the subjects made an oral response, speaking into the microphone. The subjects received 10 practice trials before participating in each block of the experiment.

Apparatus. An IBM-486 compatible microcomputer with a 14in. color VGA monitor was used for all testing. The controlling program was written in QuickBASIC 4.5, using the routines given by Graves and Bradley (1987) to achieve millisecond timing accuracy. Response times were measured as the interval between the stimulus onset and the subject's vocal response into a microphone, which caused a voice key to send an interrupt to the computer. Accuracy was also scored on line by the experimenter.

Stimuli. The stimulus words in the experiment were FLEA, MOUSE, TURTLE, PIG, GOAT, DONKEY, BEAR, and CAMEL, in this particular size ordering. Before the subjects performed the referent size- selection task, they were informed of this ordering. The stimuli were always presented in pairs, one above the other in the center of the computer screen.

There were two tasks in the experiment: the referent size-selection task and the color-selection task. In each of these tasks, there were two conditions: control and ignored repetition. However, because of the constraints of the referent size-selection task (i.e., that the target always be larger than the distractor), the ordering and pairing of stimuli differed between the two tasks. Given this, the ordering and pairing of stimuli will be presented separately for each task.

The stimulus assignment was straightforward for the colorselection task. All the stimuli occurred equally often as targets and as distractors and on the top and the bottom of the display. On a control trial, all four items making up a trial were different. The only change on ignored repetition trials was that the distractor on the prime trial matched the target on the probe trial.

The stimulus assignment was more complex for the referent sizeselection task. The complications arose primarily because of the special statuses of FLEA and CAMEL. Specifically, because FLEA is the smallest animal in the set, it can never serve as a target. Conversely, because CAMEL is the largest animal, it can never serve as a distractor. This caused a special challenge on ignored repetition trials, where an item must serve as both a distractor on the prime trial and a target on the probe trial. These constraints were dealt with in the following manner.

In the control condition of the size-selection task, all stimuli other than FLEA and CAMEL appeared equally often as targets and as distractors. Because it could never be a target, FLEA served as a distractor twice as often as the other stimuli. For the converse reason, CAMEL served as a target twice as often as the other stimuli. In all other respects, stimulus assignment in the control condition of the size-selection task matched that of the color-selection task.

In the ignored repetition condition, all the stimulus words appeared a total of 8 times as prime distractors, except for the words CAMEL and FLEA, which never appeared as prime distractors. The word CAMEL appeared a total of 12 times as the prime target, whereas the words BEAR, DONKEY, and GOAT each appeared a total of 8 times as prime targets. The words PIG and TURTLE appeared 6 times as prime targets, and MOUSE and FLEA did not appear at all as prime targets. The word FLEA appeared as the probe distractor 12 times, whereas MOUSE, TURTLE, and PIG each appeared 8 times as probe distractors. The words GOAT and DONKEY each appeared 6 times as probe distractors, whereas CAMEL and BEAR did not appear at all as probe distractors. Finally, all the stimuli appeared a total of 8 times as probe targets, except for FLEA and CAMEL, which never appeared as probe targets. Once again, this unequal assignment of stimuli was made necessary by the constraints for the referent size-selection task in general and for ignored repetition trials especially.

To make the referent size-selection and the color-selection tasks as similar as possible, one word in the pair appeared in red, and the other appeared in white, on both the prime and the probe displays. Half of the time, the target was presented in red, and half of the time it was in white.

\section{Results}

Table 1 presents the response time data for Experiment $1 \mathrm{~A}$. In both the referent size-selection and the colorselection tasks, negative priming was observed. That is, longer latencies appeared in the ignored repetition conditions, relative to their respective control conditions. Extremely large negative priming occurred in the referent size-selection task, as compared with the color-selection task. Panel A of Figure 1 presents the negative priming for Experiment $1 \mathrm{~A}$. 
Table 1

Mean Response Times (in Milliseconds), Error Rates (Proportions), and Standard Errors Across the Conditions and Tasks of Experiments $1 A$ and $1 B$

\begin{tabular}{|c|c|c|c|c|c|c|c|c|}
\hline & \multicolumn{4}{|c|}{ Ignored Repetition } & \multicolumn{4}{|c|}{ Control } \\
\hline & RT & $S E$ & ER & $S E$ & RT & $S E$ & ER & $S E$ \\
\hline \multicolumn{9}{|l|}{ Experiment $1 \mathrm{~A}$} \\
\hline Color selection & 614 & 18.52 & 011 & .00 & 579 & 15.42 & .017 & .00 \\
\hline Size selection & 1,316 & 56.17 & .066 & .01 & 1,213 & 51.79 & .084 & .01 \\
\hline \multicolumn{9}{|l|}{ Experiment 1B } \\
\hline Color selection & 579 & 11.16 & .011 & .00 & 547 & 10.62 & .005 & .00 \\
\hline Size selection & 1,211 & 51.25 & .134 & .02 & 1,110 & 42.56 & .120 & .02 \\
\hline
\end{tabular}

A $2 \times 2$ repeated measures analysis of variance (ANOVA) was conducted on the data from Experiment 1A. The variables were task (color-selection vs. referent sizeselection) and condition (control vs. ignored repetition). For this and all subsequent analyses, response times were only analyzed for correctly named probe targets that followed correctly named prime targets. We examined trimmed as well as untrimmed versions of our results, and no relevant differences were revealed. Thus, we report the untrimmed data here. With respect to errors, only error trials on probe targets that followed accurate prime responses were considered.

The latency analysis of Experiment 1A revealed significant main effects of both task $[F(1,19)=223.09$, $\left.M S_{\mathrm{e}}=39,995.93, p<.001\right]$ and condition $[F(1,19)=$ $\left.50.66, M S_{\mathrm{e}}=1,886.44, p<.001\right]$. The subjects were slower in the referent size-selection task than in the colorselection task and slower on ignored repetition trials than on control trials. The task $\times$ condition interaction was also significant $\left[F(1,19)=13.69, M S_{\mathrm{e}}=1,671.59, p<\right.$
$.001]$. The subjects responded more slowly on ignored repetition trials than on control trials, and this negative priming effect was significantly larger in the referent size-selection task than in the color-selection task.

An analogous ANOVA, performed on the errors, also revealed significant main effects of task $[F(1,19)=70.76$, $\left.M S_{\mathrm{e}}=1.80, p<.001\right]$ and condition $[F(1,19)=4.96$, $\left.M S_{\mathrm{e}}=1.58, p<.05\right]$. The significant main effects reflected the fact that the subjects made fewer errors overall in the color-selection task than in the referent sizeselection task and that they made more errors on control trials than on ignored repetition trials. This last result is especially important, inasmuch as it raises the possibility that some or all of the negative priming observed in Experiment $1 \mathrm{~A}$ was due to a speed-accuracy tradeoff. The task $\times$ condition interaction was not significant $[F(1,19)$ $\left.=1.77, M S_{\mathrm{e}}=1.19, p>.15\right]$ in the error rate analysis.

\section{EXPERIMENT 1B}

The suggestion of a speed-accuracy tradeoff, in addition to the unprecedented magnitude of negative priming observed in the referent size-selection task of Experiment $1 \mathrm{~A}$, prompted us to further examine this task. An independent replication of the experiment seemed to be a wise and cautious step. Thus, Experiment $1 \mathrm{~B}$ was a direct replication of Experiment 1A, the only change being that the data were collected in a different laboratory by a different experimenter.

\section{Method}

Subjects. Twenty volunteers from the introductory psychology class at McMaster University took part. The subjects received bonus

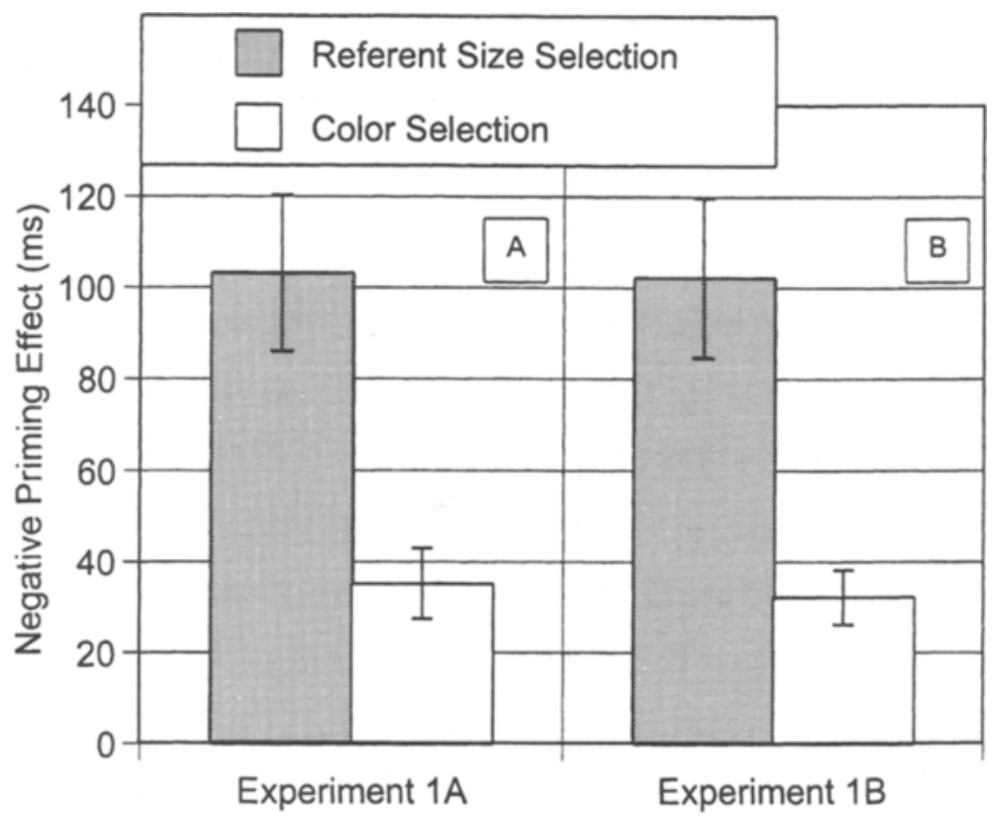

Figure 1. Negative priming scores in the referent size-selection task and the color-selection task in Experiments $1 \mathrm{~A}$ and $1 \mathrm{~B}$. Error bars reflect the standard errors of the mean. 
credit in exchange for their participation. All the subjects had normal or corrected-to-normal vision, and all had English as their first language.

Procedure. The procedures were identical to those used in Experiment 1A.

Apparatus and Stimuli. An IBM-486/DX2-66 compatible microcomputer with a 14-in. SVGA color monitor was used for all testing. In all other respects, the apparatus and stimuli were identical to those used in Experiment $1 \mathrm{~A}$.

\section{Results}

Table 1 presents the latency data for Experiment 1B. As in Experiment $1 \mathrm{~A}$, negative priming occurred in both tasks. Panel B of Figure 2 presents the negative priming for Experiment $1 \mathrm{~B}$. These negative priming scores are consistent with those obtained in Experiment 1 A. Again, the negative priming was considerably larger in the referent size-selection task than in the color-selection task. The following statistical analyses support these observations.

The data collected in Experiment 1B were subject to the same analyses as the data in Experiment 1A. The analysis of mean latencies revealed significant main effects of task $\left[F(1,19)=215.06, M S_{\mathrm{e}}=33,191.96, p<.001\right]$ and condition $\left[F(1,19)=53.68, M S_{\mathrm{e}}=1,648.75, p<.001\right]$. Again, the subjects were slower in the size-selection task than in the color-selection task and slower on ignored repetition trials than on control trials. The interaction of these variables was also significant, indicating that the observed negative priming was larger in the referent sizeselection task than in the color-selection task $[F(1,19)=$ 13.97, $\left.M S_{\mathrm{e}}=1,771.32, p<.001\right]$.

The main effect of task was also significant in the analysis performed on errors, reflecting greater errors in the referent size-selection task than in the color-selection task $\left[F(1,19)=70.30, M S_{\mathrm{e}}=5.96, p<.001\right]$. The main effect of condition and the task $\times$ condition interaction were not significant (both $F \mathrm{~s}<1$ ). Thus, in Experiment 1B, there was no worry of a speed-accuracy tradeoff being responsible for the observed negative priming effects in response time.

\section{Discussion}

The most important finding of Experiments $1 \mathrm{~A}$ and $1 \mathrm{~B}$ was the occurrence of significant negative priming in the referent size-selection task. This result is critical, because the subjects were required to attend to both targets and distractors before making their responses to targets in this task. If negative priming reflects a mechanism of selective attention (either early or late), it is not at all clear why it would result in the referent size-selection task, in which the subjects attended to and processed all items, distractors included. These results imply that the function of the negative priming mechanism is not to facilitate processing of relevant information and to prevent processing and phenomenal awareness of irrelevant information.

These findings are consistent with those of Tipper, Weaver, and Milliken (1995). In their location negative priming study, subjects were likewise required to directly compare targets and distractors. As an example, in Ex- periment 1 , the subjects were presented with a pair of circles and were asked to indicate the location of the physically larger circle. As in the present study, the subjects had to process and attend to both stimuli. Despite this fact, negative priming persisted. It should be noted that the negative priming observed by Tipper et al. (1995) was not significantly larger than the standard effect. Their study differs from ours, however, in that the targets and distractors were compared on a perceptual feature, in contrast with the semantic feature used to distinguish targets and distractors in the present study.

The second important finding of Experiments $1 \mathrm{~A}$ and $1 \mathrm{~B}$ was that not only was negative priming observed when subjects were forced to attend to distractors, it was actually enhanced. In fact, the standard negative priming effect, normally ranging from about 15 to $25 \mathrm{msec}$ (Fox, 1995), was quadrupled in the referent size-selection task. Yee, Santoro, Crawford, and Grey (1995) also observed an augmentation of negative priming, using a task remarkably similar to our referent size-selection task. They presented pairs of animal names, one in uppercase, the other in lowercase, and one in red, the other in green, with case and color completely crossed. When the subjects were asked to indicate the case of the red item, the negative priming effect was approximately $37 \mathrm{msec}$. When the subjects were asked to indicate the case of the larger animal, the negative priming effect was approximately $147 \mathrm{msec}$. Because these parallel results were observed independently, the reliability of the effect appears to be especially impressive.

This enhanced effect has practical significance. First, it provides a better medium for studying the impact of other factors on the magnitude of negative priming, not possible with the ordinarily small effect. In addition, it provides greater measurement sensitivity when dealing with populations that show reduced negative priming effects, such as obsessive-compulsives (Enright \& Beech, 1993) and schizophrenics (Beech, Powell, McWilliam, \& Claridge, 1989), or with populations that do not demonstrate the effect altogether, such as the elderly (Hasher, Stoltzfus, Zacks, \& Rympa, 1991). The precise degree of impairment can be more clearly assessed, and subtle between-population differences can emerge.

This enhanced negative priming may also have a role in shaping or differentiating theories of negative priming. Do the present findings refine existing theories or constrain their interpretation? Before we delve into the theoretical implications of the present results, a less interesting explanation of the large negative priming in the size-selection task needs exploration. Base response times in the referent size-selection task were much longer than those in the color-selection task. The large negative priming in the size-selection task might simply be an artifact of longer base response times. Perhaps a performance floor limits the magnitude of the negative priming in standard tasks. That is, the enhanced effect may only be due to an alleviation of this measurement constraint and not to any theoretically relevant feature of the task. 
We conducted several experiments to examine the alleviation-of-measurement-constraint explanation of the enhanced negative priming effect in the referent sizeselection task. In Experiments 2A and 2B, we accomplished this by examining versions of the size-selection task that allowed faster responding despite retaining the most relevant characteristic of the task (i.e., selection on the basis of the relative size or value of the items). If the enhanced negative priming is due only to an elevation of the base response time, negative priming effects in the referent size-selection task should be proportionately reduced as base response time decreases. In Experiments $3 \mathrm{~A}$ and $3 \mathrm{~B}$, we addressed the issue, using a converging strategy. In these experiments, we increased the difficulty of the color-selection task, with the goal of increasing base response time. Here, we again retained the essential characteristic of the color-selection task (i.e., selection on the basis of the color of the target item). If the alleviation of the performance floor is indeed responsible for the enhanced negative priming, increasing the base response time in the color-selection task should also yield larger negative priming.

\section{EXPERIMENTS 2A AND 2B}

The objective of Experiments 2A and 2B was to simplify the referent size-selection task so as to reduce latencies. In Experiments $1 \mathrm{~A}$ and 1B, the stimuli were not optimal, because there was some ambiguity in the size continuum. For example, we told the subjects to consider a goat to be larger than a pig in the task. Whereas some goats are larger than some pigs, the reverse is also true. Such counterexamples may have interfered with selection, thereby lengthening latencies.

To reduce response latencies, we employed stimuli that varied in value on a more objective continuum. In Experiment 2A, the stimuli were SECOND, MINUTE, HOUR, DAY, MONTH, YEAR, DECADE, and CENTURY. In Experiment $2 \mathrm{~B}$, we further simplified the continuum by using the stimuli ONE, TWO, THREE, FOUR, FIVE, SIX, SEVEN, and EIGHT.

\section{Method}

Subjects. Forty students from McMaster University participated in the experiments, half in Experiment $2 \mathrm{~A}$ and half in Experiment $2 \mathrm{~B}$. The subjects received bonus credit for their participation. All had normal or corrected-to-normal vision and English as a first language.

General. Experiments $2 \mathrm{~A}$ and $2 \mathrm{~B}$ only diverged from Experiments $1 \mathrm{~A}$ and $1 \mathrm{~B}$ in the use of different stimuli. The procedures, apparatus, and stimulus arrangements were identical.

\section{Results}

Experiment 2A. The mean latencies and the proportion of errors for Experiment $2 \mathrm{~A}$ are presented in Table 2. With regard to the referent size-selection task, the mean response time in the control condition of Experiment $2 \mathrm{~A}$ was reduced by $87 \mathrm{msec}$, as compared with the mean response time in Experiment 1B, and $190 \mathrm{msec}$, as compared with the mean response time in Experiment 1A.
Table 2

Mean Response Times (in Milliseconds), Error Rates (Proportions), and Standard Errors Across the Conditions and Tasks of Experiments $2 A$ and $2 B$

\begin{tabular}{|c|c|c|c|c|c|c|c|c|}
\hline & \multicolumn{4}{|c|}{ Ignored Repetition } & \multicolumn{4}{|c|}{ Control } \\
\hline & RT & $S E$ & ER & $S E$ & RT & $S E$ & ER & $S E$ \\
\hline \multicolumn{9}{|l|}{ Experiment $2 \mathrm{~A}$} \\
\hline Color selection & 548 & 12.90 & .004 & .00 & 529 & 12.19 & .004 & .00 \\
\hline Size selection & 1,114 & 36.20 & .122 & .02 & 1,023 & 31.96 & .080 & .01 \\
\hline \multicolumn{9}{|l|}{ Experiment 2B } \\
\hline Color selection & 539 & 12.02 & .006 & .00 & 524 & 8.77 & .005 & .00 \\
\hline Size selection & 849 & 21.44 & .087 & .02 & 793 & 19.38 & .065 & .00 \\
\hline
\end{tabular}

Negative priming again occurred in both the referent sizeselection task and the color-selection task, as is shown in panel A of Figure 2. The negative priming effects in both the size-selection task and the color-selection task were reduced slightly, relative to the negative priming obtained in the previous experiments. However, the difference in the magnitude of negative priming between the referent size-selection task and the color-selection task ( $71 \mathrm{msec})$, remained equivalent to the differences observed across the two tasks in Experiments 1A (68 msec) and $1 \mathrm{~B}(71 \mathrm{msec})$.

The analysis of the latency data revealed significant main effects of task $\left[F(1,19)=330.44, M S_{\mathrm{e}}=16,977.71\right.$, $p<.001]$ and of condition $\left[F(1,19)=63.52, M S_{\mathrm{e}}=\right.$ $946.35, p<.001]$. These results are due to longer latencies in the referent size-selection task than in the colorselection task and to longer latencies in the ignored repetition condition than in the control condition. The task $x$ condition interaction was also significant, suggesting greater negative priming in the size-selection task, relative to the color-selection task $\left[F(1,19)=36.18, M S_{\mathrm{e}}=\right.$ $705.57, p<.001]$.

The complementary analysis on errors revealed a significant main effect of task $\left[F(1,19)=94.26, M S_{\mathrm{e}}=\right.$ $3.40, p<.001]$ and a significant main effect of condition $\left[F(1,19)=6.63, M S_{\mathrm{e}}=1.70, p<.02\right]$. These results indicate that the subjects made more errors in the referent size-selection task than in the color-selection task and that they made more errors on ignored repetition trials than on control trials. Finally, the task $\times$ condition interaction was also significant, reflecting that, even in terms of errors, there was more negative priming in the referent sizeselection task than in the color-selection task $[F(1,19)=$ $\left.6.63, M S_{\mathrm{e}}=1.70, p<.02\right]$.

Experiment 2B. The mean latencies and the proportion of errors for Experiment 2B are also presented in Table 2. The mean latency in the control condition of the size-selection task for Experiment 2B was further reduced by $230 \mathrm{msec}$ relative to the mean latency in the control condition of the referent size-selection task of Experiment $2 \mathrm{~A}$. The disparity in the negative priming scores for the referent size-selection task, relative to the colorselection task, for Experiment $2 \mathrm{~B}(42 \mathrm{msec})$ was smaller than the differences obtained in previous experiments.

Statistical analysis of the latency data revealed significant main effects of task $\left[F(1,19)=372.09, M S_{\mathrm{e}}=\right.$ 


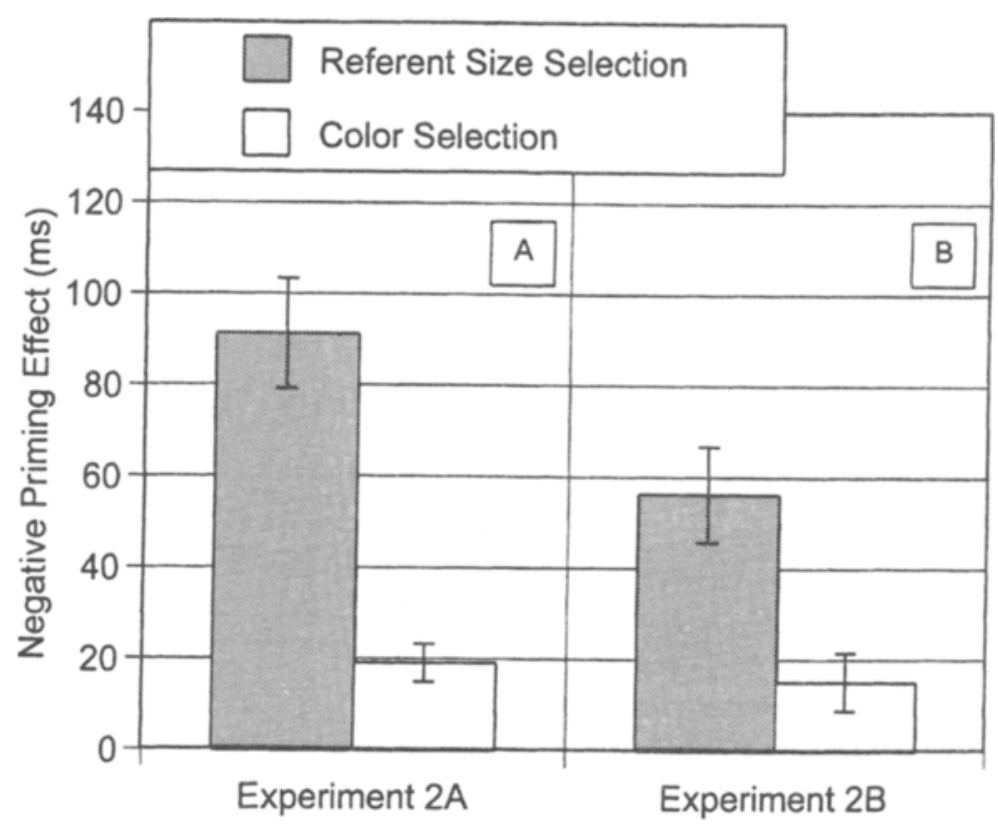

Figure 2. Negative priming scores in the referent size-selection task and the color-selection task in Experiments $2 \mathrm{~A}$ and 2B. Error bars reflect the standard errors of the mean.

$4,498.64, p<.001]$ and condition $[F(1,19)=33.21$, $\left.M S_{\mathrm{e}}=761.00, p<.001\right]$. These findings reflect longer latencies in the referent size-selection task than in the color-sclection task and for ignored repetition trials, as compared with control trials. The condition $\times$ task interaction was also significant, reflecting greater negative priming in the referent size-selection task than in the color-selection task $\left[F(1,19)=11.25, M S_{\mathrm{e}}=754.67\right.$, $p<.005]$. The parallel $2 \times 2$ ANOVA performed on errors revealed a significant main effect of task $[F(1,19)=50.54$, $\left.M S_{\mathrm{e}}=3.68, p<.001\right]$. Once again, this finding was due to there being more errors in the referent size-selection task than in the color-selection task. No other effects were significant.

\section{Discussion}

Of primary importance, we again observed negative priming when subjects were required to attend to and process distractors deeply. These results replicate the pattern found in Experiments 1A and 1B. As in Experiments $1 \mathrm{~A}$ and $1 \mathrm{~B}$, a selective attention account of negative priming (either early or late) seems to be implausible, given these findings.

The modifications in Experiments 2A and 2B were successful in decreasing latencies. Although the negative priming in the size-selection tasks remained considerably greater than the standard effect, the scores were smaller than those observed in Experiments 1A and 1B. Considering only the referent size-selection data, it appears as though decreases in base response time are accompanied by reductions in negative priming. However, examining the disparity in the negative priming scores for the refer- ent size-selection tasks and the color-selection tasks, the difference decreased slightly in Experiment $2 \mathrm{~B}$ and not at all in Experiment 2A. Negative priming effects vary with stimuli and with subject groups. Given that the colorselection and referent size-selection tasks are matched on these variables, considering the referent size-selection task, relative to the color-selection task, seems to be the most appropriate examination.

\section{EXPERIMENTS 3A AND 3B}

The purpose of Experiments $3 \mathrm{~A}$ and $3 \mathrm{~B}$ was to provide converging evidence with the results of Experiments $2 \mathrm{~A}$ and $2 \mathrm{~B}$. It was our intention to rule out the alleviation-of-measurement-constraint argument as the sole explanation for the enhanced negative priming in the referent size-selection task. In Experiments $3 \mathrm{~A}$ and $3 \mathrm{~B}$, rather than decreasing latencies in the size-selection task, our objective was to increase latencies in the colorselection task. This was achieved by presenting items interleaved, instead of one on top of the other, as had been done in previous experiments (Milliken \& Joordens, 1996; Milliken et al., 1998). Experiment 3B differed from Experiment $3 \mathrm{~A}$ in that target and distractor colors were more similar. If the magnitude of negative priming is a function of base response times, negative priming in a more difficult version of a color-selection task should increase as base response latencies increase.

\section{Method}

Subjects. Thirty-eight undergraduate students at McMaster University participated in the experiments, 20 in Experiment $3 \mathrm{~A}$ and 18 
in Experiment 3B. All received bonus credit in their introductory psychology class and had normal or corrected-to-normal vision.

Procedure. All the subjects performed both the standard and the difficult versions of the color-selection task. The order of tasks was counterbalanced across subjects. The procedure for the standard color-selection task was the same as that described in previous experiments. For the difficult version, the only change was that targets and distractors were interleaved in the center of the computer screen (e.g., "*TCUARMTELLE*). In Experiment 3A, targets were red and distractors were white, whereas in Experiment $3 B$, targets were red and distractors were brown.

Apparatus and Stimuli. The apparatus, stimuli, and stimulus arrangements were the same as in the color-selection task of Experiment $1 B$. Because the length of the stimuli varied, all the stimuli were made to have an eight-character length by adding asterisks on either side of the word in the difficult version. In the difficult color-selection task, targets and distractors appeared equally in the position slightly to the right of the display or slightly to the left, on both the prime and the probe.

\section{Results}

Experiment 3A. Latencies and proportion of errors for Experiment 3A appear in Table 3. In the difficult color-selection task, the mean latency in the control condition was increased by $242 \mathrm{msec}$, relative to that of the standard color-selection task. The negative priming scores, however, did not differ, despite this change in base response time. Panel A of Figure 3 presents the negative priming. These observations are confirmed by the following statistical analyses.

The analysis on the latencies in Experiment $3 \mathrm{~A}$ revealed significant main effects of task $[F(1,19)=83.76$, $\left.M S_{\mathrm{e}}=14,257.32, p<.001\right]$ and condition $[F(1,19)=$ $\left.32.40, M S_{\mathrm{e}}=540.81, p<.001\right]$. The main effect of task reflects longer latencies in the difficult color-selection task than in the standard color-selection task. The main effect of condition was due to longer latencies on ignored repetition trials than on control trials (i.e., negative priming). Importantly, the task $\times$ condition interaction was not significant $[F(1,19)<1]$. This indicates that, although responses were much slower in the difficult version of the task, the negative priming was not significantly larger, as would be predicted by the alleviationof-measurement-constraint account.

The analysis of errors revealed only a significant main effect of task $\left[F(1,19)=9.50, M S_{\mathrm{e}}=0.53, p<.025\right]$. The subjects made more errors in the difficult color-selection

\section{Table 3}

Mean Response Times (in Milliseconds), Error Rates (Proportions), and Standard Errors Across the Conditions and Tasks of Experiments $3 A$ and $3 B$

\begin{tabular}{|c|c|c|c|c|c|c|c|c|}
\hline & \multicolumn{4}{|c|}{ Ignored Repetition } & \multicolumn{4}{|c|}{ Control } \\
\hline & RT & $S E$ & ER & $S E$ & RT & $S E$ & ER & $S E$ \\
\hline \multicolumn{9}{|l|}{ Experiment 3A } \\
\hline Standard selectio & 566 & 13.23 & .004 & .00 & 539 & 9.42 & .005 & .00 \\
\hline Difficult selec & 813 & 34.12 & .015 & .00 & 781 & 35.16 & .016 & .01 \\
\hline \multicolumn{9}{|l|}{ Experiment $3 \mathrm{~B}$} \\
\hline Standard selectio & 554 & 9.94 & .008 & .00 & 535 & 10.49 & .009 & .00 \\
\hline Difficult selection & 826 & 23.00 & .029 & .01 & 802 & 26.16 & .022 & .01 \\
\hline
\end{tabular}

task than in the standard color-selection task. This effect did not interact with condition $[F(1,19)<1]$.

Experiment 3B. Latencies and errors for Experiment 3B also appear in Table 3. Relative to the mean latency in the control condition of the standard colorselection task, that of the difficult color-selection task was increased by $267 \mathrm{msec}$. Again, the size of the negative priming effect did not differ between tasks. Panel B of Figure 3 presents the negative priming for Experiment 3B.

Analysis of the data collected in Experiment 3B revealed results that parallel those in Experiment $3 \mathrm{~A}$. The main effect of condition was significant with longer response times on ignored repetition trials than on control trials $\left[F(1,17)=18.52, M S_{\mathrm{e}}=459.77, p<.001\right]$, indicating reliable negative priming. The main effect of task was also significant, with slower responses on the difficult version of the task than on the standard version $[F(1,17)=$ $132.74, M S_{\mathrm{e}}=9,818.48, p<.001$. These two factors did not interact $[F(1,17)<1]$. Although latencies increased in the difficult color-selection version of the task, the negative priming did not.

The complementary analysis of errors revealed only a main effect of task $\left[F(1,17)=9.50, M S_{\mathrm{e}}=1.07, p<.010\right]$. This reflects greater errors in the difficult color-selection task than in the standard color-selection task. This effect did not interact with condition $[F(1,17)<1]$.

\section{Discussion}

The manipulations in Experiments $3 \mathrm{~A}$ and $3 \mathrm{~B}$ were successful in increasing latencies in the difficult colorselection tasks, relative to the standard color-selection tasks. Despite dramatic increases in base response times, negative priming was essentially unaffected by the manipulation. Although we had intended to increase latencies further in Experiment 3B, the base response time was not significantly boosted beyond that achieved in Experiment 3A. Experiment 3B replicated the critical pattern of negative priming obtained in Experiment $3 \mathrm{~A}$.

In Experiments 2A, 2B, 3A, and 3B, negative priming was only negligibly affected by base response times. Despite its contribution to the enhanced negative priming in the referent size-selection tasks, the alleviation-ofmeasurement-constraint explanation is by no means the whole story. ${ }^{1}$ The large negative priming observed in the referent size-selection tasks occurred mostly because of a unique feature of the task, rather than as an artifact of lengthened latencies.

\section{GENERAL DISCUSSION}

The findings can be summarized quite briefly. Negative priming persists even when the typical procedure is modified so that subjects are required to attend to both the targets and the distractors. Not only does it persist, the magnitude of the effect actually increases about fourfold. This enhanced effect is not solely due to an alleviation of a performance floor. 


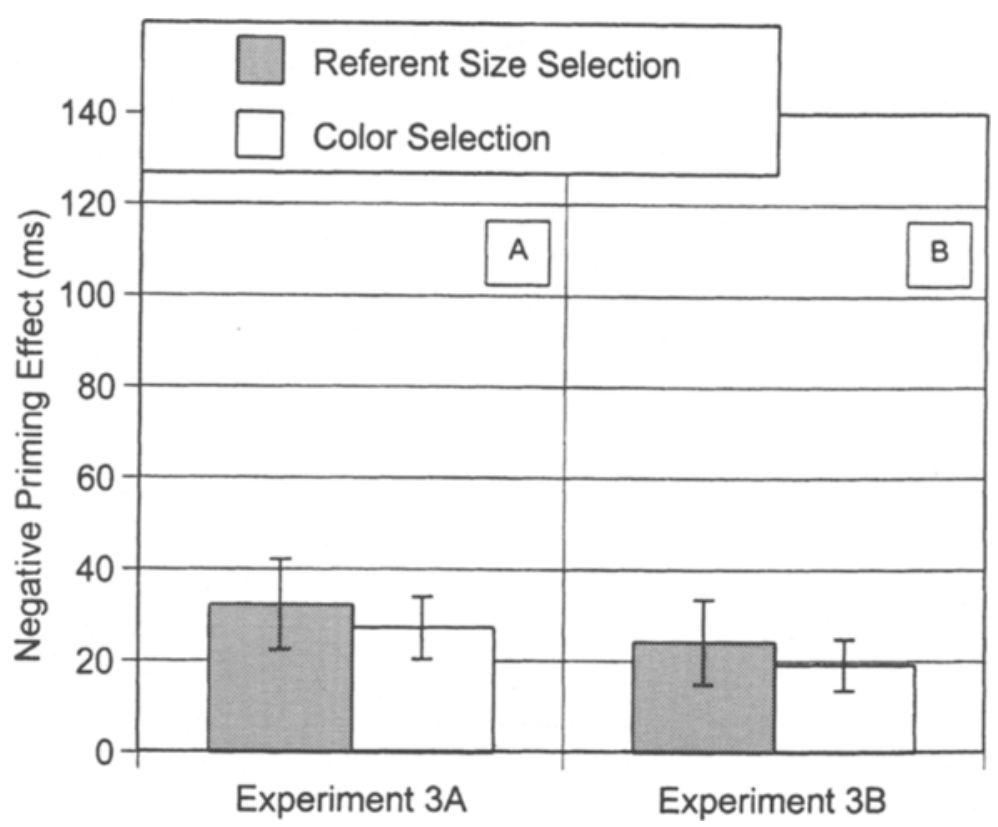

Figure 3. Negative priming scores in the difficult color-selection task and the standard color-selection task in Experiments 3A and 3B. Error bars reflect the standard errors of the mean.

The discussion is composed of two sections. The first focuses on the implications of our results for current theories of negative priming. The second discusses the primary issue brought to light by the study. Specifically, our results question whether negative priming indeed results from selective attention, given that it occurs even when both stimuli are attended.

\section{Implications for Current Theories of Negative Priming}

Distractor inhibition. The most recent inhibition account of negative priming is the computational model of Houghton and Tipper (1994). According to this account, initially both the target's and the distractor's representations receive activation. Target selection occurs when the activation level of the target's representation is a given quantity greater than that of the distractor. If left unchecked, both the target and the distractor would gain activation at similar rates. To separate the target from the distractor, a mechanism is engaged that selectively inhibits the distractor's representation. As the distractor continues to receive bottom-up activation, the net effect of the inhibition is to slow the distractor's activation gain, relative to that of the target, facilitating selection. Upon target selection, however, the bottom-up source of activation is removed (i.e., the physical representations disappear). The temporary persistence of the inhibition of the distractor's representation results in its being forced below baseline. If the distractor occurs as a subsequent target, it takes longer to gain target levels of activation.

Can this model account for our findings? Our data seem most problematic for the inhibition view of negative prim- ing, which is based on notions of selective attention. A recent study (Houghton, Tipper, Weaver, \& Shore, 1996), however, examined the related issue of distractor salience and may address our results. Houghton et al. demonstrated that, similar to the findings in the present study, larger negative priming resulted when distractors were made more salient. Their notion was that "... inhibitory systems may be energized by that which they inhibit" (p. 140). The distractor in their study was perceptually very salient, attracting attention. If this interpretation purports that the greater inhibition, which gives rise to the large negative priming, serves to keep the highly salient distractors from attentional focus and awareness, then the present findings are at odds with this view of negative priming. If their view, however, is that the greater inhibition serves to suppress responses to distractors, although both targets and distractors are attended and processed, then the inhibition view can account for the data.

Recently, Tipper and his colleagues appear to be favoring the latter interpretation with their espousal of the concept of selection for action. This is evident in the following. "In short, we ask: How is it that individual responses can be selectively controlled by particular stimuli in the presence of competing inputs?" (Houghton et al., 1996, p. 140). Despite this endorsement of selective responding or selection for action, this distinction is not consistently and clearly made. In the immediately preceding sentence, the authors stated that "we consider the problem faced by such an organism to be a problem of selective attention." Relating negative priming to selective attention and selective responding interchangeably, particularly if it is not explicit whether an early or a late 
attentional mechanism is proposed, confuses the issue and potentially produces erroneous expectations (i.e., that negative priming results from blocking distractors from attentional focus). Given the present data and that of others (Tipper et al., 1995; Yee et al., 1995), only the view that negative priming results from selective responding or selection for action remains plausible.

Episodic retrieval. The episodic-retrieval account provides an alternative explanation of negative priming (Neill \& Valdes, 1992; Neill, Valdes, Terry, \& Gorfein, 1992). This view maintains that distractors and targets undergo different encoding processes. According to variations of this account, distractors either are encoded with "do not respond" tags or are encoded without any response information at all. In contrast, targets are encoded with "respond" tags or with specific response instructions. The view posits that when subjects make their selection of targets on probe trials, they gather additional information about the item by retrieving prior processing episodes containing the current target item. When a word that was previously encoded as a distractor, with the accompanying response information, appears as a target on the following trial, this backward-acting retrieval process produces information that conflicts with the present status of the item. A delay (i.e., negative priming) ensues, during which subjects resolve the ambiguity created by the response competition (i.e., "do not respond" on the first trial and "respond" on the second trial). The additional assumption that the last episode is the most temporally discriminable, thus the most likely retrieved, is required for this explanation to adequately account for the findings.

Does the episodic-retrieval account of negative priming explain our data? It seems to be reasonable to assume that our referent size-selection task requires deeper processing of distractors on both prime and probe trials. It follows that the prime-trial memory trace for the distractor should be significantly strengthened. Therefore, from the episodic-retrieval view, the probability of recalling the prime episode in the ignored repetition condition of the size-selection task is greater than that probability in the color-selection task. As a result, this confusion or response competition will occur on a greater proportion of ignored repetition trials in the referent size-selection task, which should yield larger negative priming. Thus, the episodic-retrieval account provides a straightforward explanation of the findings in the present study.

Feature mismatch. One of the earliest theories of negative priming was the code-coordination theory (Lowe, 1979) or, more recently, the feature mismatch theory (Park \& Kanwisher, 1994). The basic notion is that negative priming results from the availability of conflicting information about a probe target from its previous occurrence on the prime. Whereas the episodic-retrieval account focuses on the retrieval of incompatible response information, perceptual mismatch theory centers on the retrieval of mismatching perceptual information. In most negative priming experiments, identifying features (e.g., colour, font, size) or location (e.g., above, below, right) of the stimulus indicate target status. Consider the colorselection task, in which subjects were asked to read red words and to ignore white words. On ignored repetition trials, the distractor on the prime trial appears in white, then in red, as the target on the probe trial. Perceptual features of the item-the color being of particular importance in this instance - are different on the prime and probe. This mismatch from sequential trials introduces some ambiguity, purportedly delaying responding on ignored repetition trials, relative to control trials.

A perceptual mismatch account of our results seems improbable. Because the selection criterion was referent size and not a perceptual feature, on some ignored repetition trials the target item on the probe was perceptually identical to its prior presentation on the prime. Juan Lupiáñez Castillo (personal communication), however, convinced us that semantic mismatch in our size-selection task might account for the data. Consider that, on an ignored repetition trial, an animal deemed to be small on the prime becomes the larger animal in the pair on the probe. This mismatch in size assignment could hamper target selection on the probe. In fact, semantic mismatch may be even more difficult to resolve, explaining the enhanced negative priming.

Summary. It appears that all the accounts of negative priming are or can be made consistent with our results. The present findings, however, constrain and refine interpretations and predictions. Furthermore, the augmented effect may prove to be useful in differentiating these theories. For example, to confirm the mismatch view, it must be shown that negative priming is eliminated when there is no mismatch in the presentation of the critical item on the prime and the probe. With a small (i.e., $15-25 \mathrm{msec}$ ) and somewhat unstable effect, eliminating negative priming in the desired condition of the standard task is not especially impressive and could be disputed on the grounds of the inconsistency of negative priming. If a 100 -msec effect, which has been demonstrated to occur consistently, is abolished through the elimination of mismatch, however, the evidence is far less contestable.

\section{Selective Attention and Negative Priming}

Our primary aim was to directly examine the link between selective attention and negative priming. This was achieved by ensuring that both targets and distractors were attended to, were processed deeply, and reached phenomenal awareness on all trials. Negative priming persisted and actually increased nonetheless, suggesting that it is not produced by selective attention, either early or late. These findings, along with those of Tipper et al. (1995), Yee et al. (1995), Milliken and Joordens (1996), and Milliken et al. (1998), are inconsistent with the claim that stimuli are responded to more slowly in the ignored repetition condition as a result of being previously 
ignored or processed without attention. Negative priming does not result from a mechanism that limits deep processing and phenomenal awareness to relevant items only.

\section{REFERENCES}

Beech, A., Powell, T., McWilliam, J., \& Claridge, C. (1989). Evidence of reduced "cognitive inhibition" in schizophrenia. British Journal of Clinical Psychology, 28, 110-116.

Broadbent, D. E. (1958). Perception and communication. London: Pergamon.

ChiapPe, D. L., \& MaCLeod, C. M. (1995). Negative priming is not task bound: A consistent pattern across naming and categorization tasks. Psychonomic Bulletin \& Review, 2, 364-369.

Dalrymple-Alford, E. C., \& Budayr, B. (1966). Examination of some aspects of the Stroop colour-word test. Perceptual \& Motor Skills, 23, 1211-1214.

Deutsch, J. A., \& Deutsch, D. (1963). Attention: Some theoretical considerations. Psychological Review, 70, 80-90.

DrIVER, J., \& BAYLIS, G. C. (1993). Cross-modal negative priming and interference in selective attention. Journal of Experimental Psychology: Human Perception \& Performance, 15, 45-48.

ENRIGHT, S. J., \& BEECH, A. R. (1993). Reduced cognitive inhibition in obsessive-compulsive disorder. British Journal of Clinical Psychology, 32, 67-74.

Fox, E. (1995). Negative priming from ignored distractors in visual selection: A review. Psychonomic Bulletin \& Review, 2, 145-173.

Graves, R., \& BRADLEY, R. (1987). Millisecond interval timer and auditory reaction time programs for the IBM PC. Behavior Research Methods, Instruments, \& Computers, 19, 30-35.

Hasher, L., Stoltzfus, E. R., Zacks, R. T., \& Rympa, B. (1991). Age and inhibition. Journal of Experimental Psychology: Learning, Memory, \& Cognition, 17, 163-169.

Houghton, G., \& TIPPER, S. P. (1994). A model of inhibitory mechanisms in selective attention. In A. Dagenbach \& T. H. Carr (Eds.), Inhibitory mechanisms in attention, memory, and language (pp. 53 112). San Diego: Academic Press.

Houghton, G., Tipper, S. P., Weaver, B., \& Shore, D. I. (1996). Inhibition and interference in selective attention: Some tests of a neural network model. Visual Cognition, 3, 119-164.

LowE, D. G. (1979). Strategies, context, and the mechanism of response inhibition. Memory \& Cognition, 7, 382-389.

MAY, C. P., KANE, M. J., \& HASHER, L. (1995). Determinants of negative priming. Psychological Bulletin, 118, 35-54.

Milliken, B., \& JoORdenS, S. (1996). Negative priming without overt prime selection. Canadian Journal of Experimental Psychology, 50, 333-346.

Milliken, B, Joordens, S., Merikle, P., \& Seiffert, A. (1998). Selective attention: A re-evaluation of the implications of negative priming. Psychological Review, 105, 203-229.

NeILL, W. T. (1977). Inhibitory and facilitatory processes of selective attention. Journal of Experimental Psychology: Human Perception \& Performance, 3, 444-450.

NeILL, W. T., LISSNER, L. S., \& BECK, J. L. (1990). Negative priming in same-different matching: Further evidence for a central locus of inhibition. Perception \& Psychophysics, 48, 398-400.

NeILL, W. T., \& VAlDES, L. A. (1992). Persistence of negative priming: Steady state or decay? Journal of Experimental Psychology: Learning, Memory, \& Cognition, 18, 565-576.

Neill, W. T., Valdes, L. A., Terry, K. M., \& Gorfein, D. S. (1992). The persistence of negative priming: II. Evidence for episodic trace retrieval. Journal of Experimental Psychology: Learning. Memory, \& Cognition, 18, 993-1000.

PARK, J., \& KaNWISHER, N. (1994). Negative priming for spatial location: Identity mismatching, not distractor inhibition. Journal of Experimental Psychology: Human Perception \& Performance, 20, 613-623.

TIPPER, S. P. (1985). The negative priming effect: Inhibitory priming by ignored objects. Quarterly Journal of Experimental Psychology, 37A, 571-590.

TIPPER, S. P., \& BAYLIS, G. C. (1987). Individual differences in selective attention: The relation of priming and interference to cognitive failure. Personality \& Individual Differences, 8, 667-675.

TIPPER, S. P., \& DrIVER, J. (1988). Negative priming between pictures and words in a selective attention task: Evidence for semantic processing of ignored stimuli. Memory \& Cognition, 16, 64-70.

TipPer, S. P., MacQueEn, G. M., \& Brehaut, J. C. (1988). Negative priming between response modalities: Evidence for the central locus of inhibition in selective attention. Perception \& Psychophysics, 43, 45-52.

TiPPER, S. P., Weaver, B., \& Milliken, B. (1995). Spatial negative priming without mismatching: Comment on Park and Kanwisher (1994). Journal of Experimental Psychology: Human Perception \& Performance, 21, 1220-1229.

YEE, P. L. (1991). Semantic inhibition of ignored words during a figure classification task. Quarterly Journal of Experimental Psychology, 43A, 127-152.

Yee, P. L., Santoro, K. E., Crawford, T., \& Grey, A. (1995, November). Negative priming effects under conceptual target selection conditions. Poster presented at the 36th Annual Meeting of the Psychonomic Society, Los Angeles.

\section{NOTE}

1. Correlating the mean response times in the control conditions with the negative priming scores in that particular task for each subject across all the experiments in the study also did not support the alleviation-ofmeasurement-constraint account. Longer latencies were not associated with larger negative priming scores.

(Manuscript received October 20, 1997; revision accepted for publication April 6, 1998.) 